\title{
Synthesis of a High Step-up Bipolar Voltage Multiplier Using Level Shift Drivers
}

\author{
Kanji Abe ${ }^{1+}$, Ichirou Oota ${ }^{2}$, Wang Lok Do ${ }^{1}$ and Kei Eguchi ${ }^{1}$ \\ ${ }^{1}$ Fukuoka Institute of Technology \\ ${ }^{2}$ National Institute of Technology, Kumamoto College
}

\begin{abstract}
In non-thermal food processing by utilizing an underwater shockwave, high voltage multiplier is required to generate an underwater shockwave. The conventional high voltage multipliers can generate a high voltage in a simple circuit configuration. However, the operation speeds of the conventional voltage multipliers are slow. Therefore, a novel high voltage multiplier for non-thermal food processing is proposed in this paper. Unlike the conventional multipliers, the proposed voltage multiplier is controlled by high speed four phase pulses generated by a level shift drivers which have bipolar input terminals. Therefore, the proposed voltage multiplier can generate a high voltage at high speed. Furthermore, the proposed voltage multiplier can achieve the smaller number of the circuit components than that of the conventional voltage multiplier. To confirm the validity of the proposed multiplier, experiments are performed concerning the proposed multiplier assembled with commercially available components.
\end{abstract}

Keywords: cockcroft-walton multipliers, high voltage multipliers, high speed multipliers, non-thermal food processing, step-up voltage multipliers.

\section{Introduction}

The cooking process, which is generally used for processing foods, has a problem that nutrients of foods are destroyed by heating. Therefore, a non-thermal food processing attracts much researchers' attention [1], because it does not destroy the nutrients of foods. Among them, we focus on the non-thermal food processing by utilizing an underwater shockwave [2], because it can be realized at relatively low cost and safety. This non-thermal food processing system mainly consists of a pressure vessel and a high voltage multiplier which can generate more than $3.5 \mathrm{kV}$. In this non-thermal food processing, the high voltage multiplier has to discharge a high voltage repeatedly so that the underwater shockwave is generated in the pressure vessel. A Cockcroft-Walton voltage multiplier (CWVM) [3] is famous as a traditional voltage multiplier. However, according to the increase of the number of stages, the output voltage of the CWVM begins to sag. Furthermore, the response speed of the CWVM is slow, because the diode switch of the CWVM is controlled by a sinusoidal waveform supplied by an electric outlet. In past studies, Iqbal [4] proposed a hybrid symmetrical CWVM. In the hybrid symmetrical CWVM, both the voltage efficiency and the transient response speed are improved from the CWVM owing to a transformer and a symmetrical structure. However, the hybrid symmetrical CWVM is heavy and expensive due to the transformer. To realize a low-cost voltage multiplier, Eguchi et al. [5-6] suggested a parallel-connected high voltage multiplier (PCVM). Owing to the parallel-connected structure, the PCVM can generate a high voltage at high speed without a transformer. However, there is still room for improvement in this multiplier.

In this paper, a high step-up bipolar voltage multiplier using level shift drivers is proposed for nonthermal food processing by utilizing an underwater shockwave. The proposed multiplier consists of three positive multiplier blocks and three negative multiplier blocks. In the proposed multiplier, one positive

\footnotetext{
${ }^{+}$Corresponding author. Tel.: +81-92-606-3137; fax: +81-92-606-0726.

E-mail address: mam15001@bene.fit.ac.jp
} 
multiplier and one negative multiplier also work as AC-DC rectifiers. The other multipliers are connected via the driver circuit in parallel. To generate a high voltage at high speed, the parallel-connected multipliers are controlled by high speed four phase pulses. The amplitude of the four phase pulses is higher than the input voltage owing to the AC-DC rectifier. Owing to these features, the proposed voltage multiplier can achieve not only high voltage efficiency but also high speed operation. Furthermore, the number of the circuit components of the proposed voltage multiplier is smaller than that of the conventional voltage multiplier. To confirm the validity of the proposed multiplier, experiments are performed concerning the proposed multiplier assembled with commercially available components.

\section{Circuit configuration}

\subsection{Conventional voltage multiplier}

Figure 1 shows the Cockcroft-Walton voltage multiplier (CWVM) with $N$ stages $(N=1,2, \ldots)$. To generate a high voltage, the CWVM is controlled by a sinusoidal waveform supplied by a commercial power source.

The output voltage of the CWVM is expressed as

$$
V_{\text {out }}=2 N\left(V_{\text {in }}-V_{\text {th }}\right) . \quad(N=1,2, \ldots)
$$

In (1), $V_{\text {in }}$ means an input AC voltage and $V_{t h}$ means the threshold voltage of the diode switch.

As Figure 1 shows, the CWVM can generate a high voltage in a simple circuit configuration. However, since CWVM is controlled by a sinusoidal waveform supplied by an electric outlet, its response speed is slow. Furthermore, the impedance of each capacitor is high due to the low frequency of the input voltage, so the voltage efficiency of the CWVM is low.

As Figure 1 shows, one stage of the CWVM has four components: two capacitors and two diode switches. According to (1), the CWVM must be more than 13 stages for generating more than $3.5 \mathrm{kV}$. Therefore, to generate an underwater shockwave in the non-thermal food processing, the CWVM with 13 stages requires $52(=4 \times 13)$ components: 26 capacitors and 26 diode switches.

\subsection{Proposed voltage multiplier}

Figure 2 shows the proposed high step-up bipolar voltage multiplier using level shift drivers. As Figure 2 shows, the proposed voltage multiplier consists of three blocks: an AC-DC rectifier block, a driver circuit block and a parallel-connected bipolar voltage multiplier (PCBVM) block. The new features of the proposed voltage multiplier are as follows: (1) to generate high voltage pulses, the AC-DC rectifier block has two voltage multipliers, which are a positive and a negative CWVM with symmetrical structure. (2) The driver circuit has four switches. Figure 2 also shows setting of clock pulses for these switches. By controlling these switches at high speed, high speed four phase pulses are generated. (3) The PCBVM block has four voltage multipliers. By operating PCBVM blocks by the four phase pulses generated by the driver circuit, the proposed voltage multiplier can generate an output voltage at high speed and (4) to offer high voltage efficiency, a max operation circuit and a min operation circuit are connected to output terminals. Owing to these new features, the proposed voltage multiplier realizes high voltage efficiency and high speed operation.

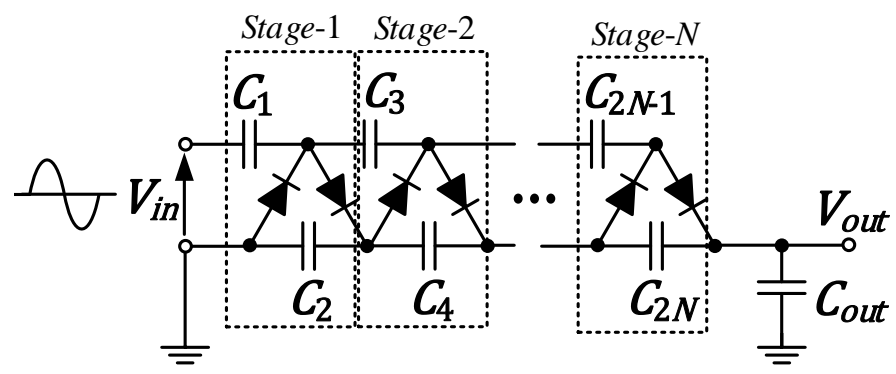

Fig. 1: Cockcroft-Walton voltage multiplier. 


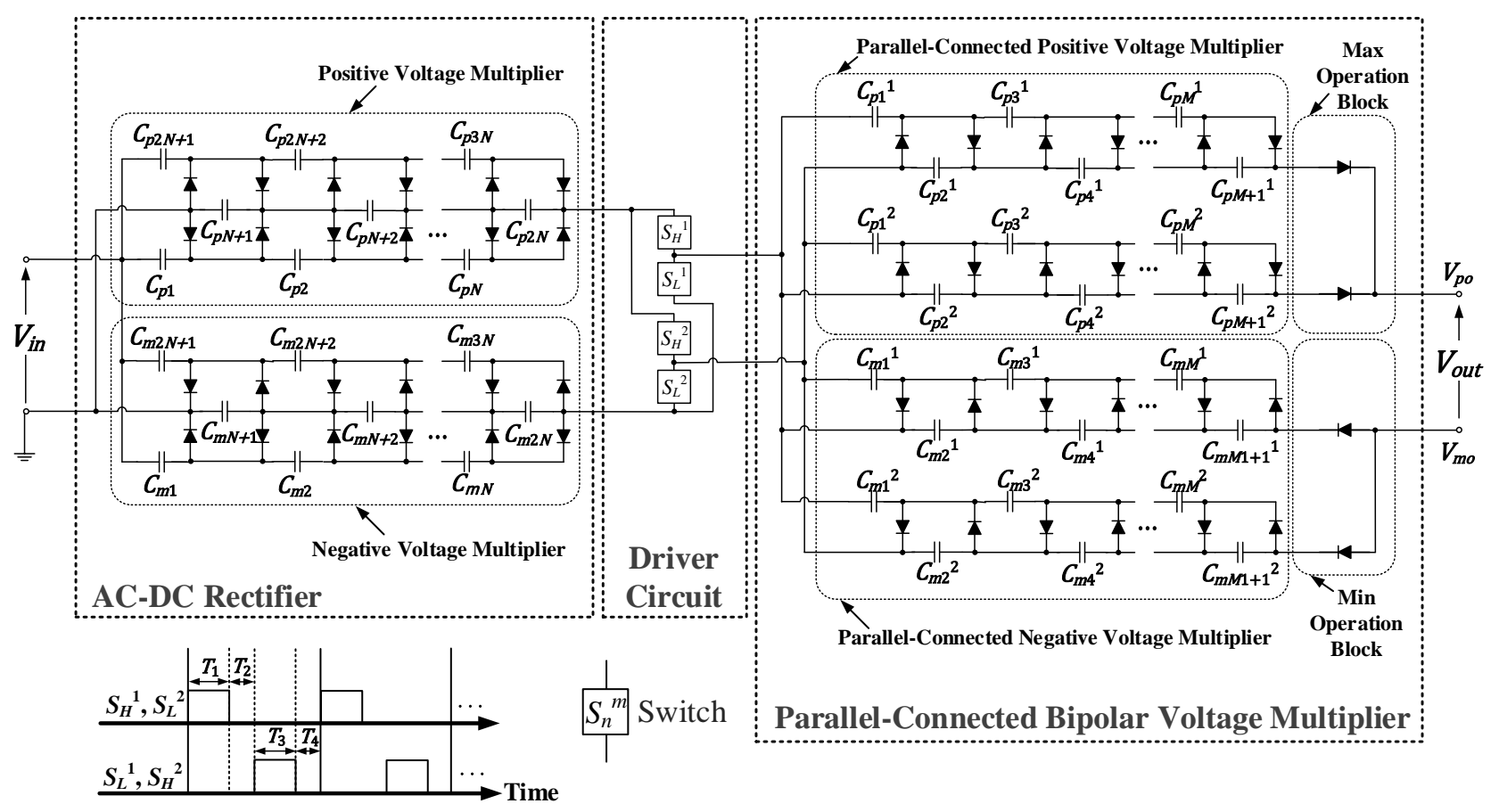

Fig. 2: Proposed voltage multiplier.

The output voltage of the proposed voltage multiplier is expressed as the following equation:

$$
\begin{aligned}
V_{\text {out }}= & V_{p o}-V_{\text {no }} \\
= & 4 N \cdot(4 M+1) \cdot V_{\text {in }} \\
& -\{(4 M+1) \cdot(4 N+1)+1\} \cdot V_{t h},
\end{aligned}
$$

Where $V_{p o}=V_{m o} \cong(4 M+1) \cdot V_{c w}-(2 M+1) \cdot V_{t h}$ and $V_{c w}=2 N\left(V_{i n}-V_{t h}\right)$

In (2), $V_{i n}$ is the an input AC voltage, $V_{t h}$ is the threshold voltage of the diode switch, $N(=1,2, \ldots)$ is the number of stages of the AC-DC rectifier block, $M(=1,2, \ldots)$ is the number of stages of the PCBVM block, $V_{p o}$ is the positive output voltage of the PCBVM block, $V_{m o}$ is the negative output voltage of the PCBVM block and $V_{c w}$ is the output voltage of the AC-DC rectifier block. As Figure 1 shows, 1 stage of the AC-DC rectifier has 14 components; 6 capacitors and 8 diodes switches. Then, one stage of the PCBVM has 16 components; 8 capacitors and 8 diodes switches. Furthermore, the driver circuit consists of only four switches, and max/min operation blocks consist of only four diode switches. By adjusting the number of stages of the AC-DC rectifier to 1 stage and the PCBVM to the following stages: $M_{p}=2$ and $M_{m}=1$, the proposed voltage multiplier can generate more than $3.5 \mathrm{kV}$. Therefore, to generate an underwater shockwave in the non-thermal food processing, the proposed voltage multiplier requires 46 components: the AC-DC rectifier $(N=1)$ requires 14 components, the driver circuit requires 4 switches, the PCBVM block $\left(M_{p}=2\right.$ and $M_{m}=1$ ) requires 24 components, max operation block requires 2 components and min operation block requires 2 components. Table 1 shows the comparison of the number of circuit components between the conventional CWVM and the proposed voltage multiplier. As table 1 shows, the proposed multiplier can offer not only high voltage efficiency and high speed operation but also the small number of circuit components.

\section{Experiments}

To clarify the validity of the proposed multiplier, experiments are performed. The number of stages of the conventional CWVM was set to thirteen, and that of the proposed voltage multiplier was set to $M_{p}=2$ and $M_{m}=1$. Table 2 shows the circuit components of the experimental voltage multipliers. As table 2 shows, the circuit components of the proposed voltage multiplier were set to the same components of the conventional CWVM. The experimental conditions of the conventional CWVM and the proposed voltage multiplier are as 
follows: $V_{\text {in }}=100 \mathrm{~V} @ 60 \mathrm{~Hz}, T=66.7 \mu$ s and output capacitor $C_{\text {out }}=2.2 \mu \mathrm{F}(=33 \mu \mathrm{F} / 16)$. The output capacitors was connected in series to increase its withstand voltage. In the experiments, to isolate the input source from the output load, a small transformer was connected with the experimental circuits. The inductance ratio of the primary and secondary of the transformer was set to 1:1. However, the turn ratio of the experimental transformer was 1:1.1 due to the fluctuation practically, so the experimental input voltage was about $128 \mathrm{~V}$ (= $141 \mathrm{~V} / 1.1)$. In the proposed voltage multiplier, photo MOS relays were used as switches. To drive the photo MOS relays securely, Darlington transistor arrays were used between the photo MOS relays and the microcontroller.

Figure 3 shows the measured output voltages. As Figure 3 (a) shows, the output voltage of the conventional CWVM is $2.65 \mathrm{kV}$. On the other hand, as Figure 3 (b) shows, the output voltage of the proposed voltage multiplier is $3.56 \mathrm{kV}$. From these results, the output voltage of the proposed voltage multiplier is $0.91 \mathrm{kV}$ higher than that of the conventional CWVM.

Figure 4 shows the transient response of the measured output voltages. As Figure 4 (a) shows, the rise time of the conventional CWVM is about $69.87 \mathrm{sec}$. On the other hand, as Figure 4 (b) shows, the rise time of the proposed voltage multiplier is about $4.823 \mathrm{sec}$. From these results, the rise time of the proposed voltage multiplier is more than 14 times faster than that of the conventional CWVM.

As these results show, the proposed voltage multiplier can offer not only high voltage efficiency but also high speed operation.

Table 1: Comparison of the number of circuit components between the CWVM and the proposed multiplier.

\begin{tabular}{|c|c|c|}
\hline Components & Conventional CWVM $(N=13)$ & Proposed voltage multiplier $\left(N=1, M_{p}=2, M_{m}=1\right)$ \\
\hline \hline Capacitor & 26 & 18 \\
\hline Switch & 0 & 4 \\
\hline Diode & 26 & 24 \\
\hline \hline Total & 52 & 46 \\
\hline
\end{tabular}

Table 2: Circuit components of the experimental voltage multipliers.

\begin{tabular}{|c|c|c|}
\hline \multicolumn{1}{|c|}{ Multiplier } & Component & Model \\
\hline \hline \multirow{3}{*}{ Conventional CWVM } & Capacitor & $1 \mu \mathrm{F}$ \\
\cline { 2 - 3 } & Diode switch & $1 \mathrm{~N} 4007$ \\
\hline \multirow{5}{*}{ Proposed voltage multiplier } & Capacitor of the AC-DC rectifier & $10 \mu \mathrm{F}$ \\
\cline { 2 - 3 } & Capacitor of the PCBVM & $1 \mu \mathrm{F}$ \\
\cline { 2 - 3 } & Diode switch & AQW216 \\
\cline { 2 - 3 } & Switch & TD62004APG \\
\cline { 2 - 3 } & Driver IC & PIC12F1822 \\
\cline { 2 - 3 }
\end{tabular}

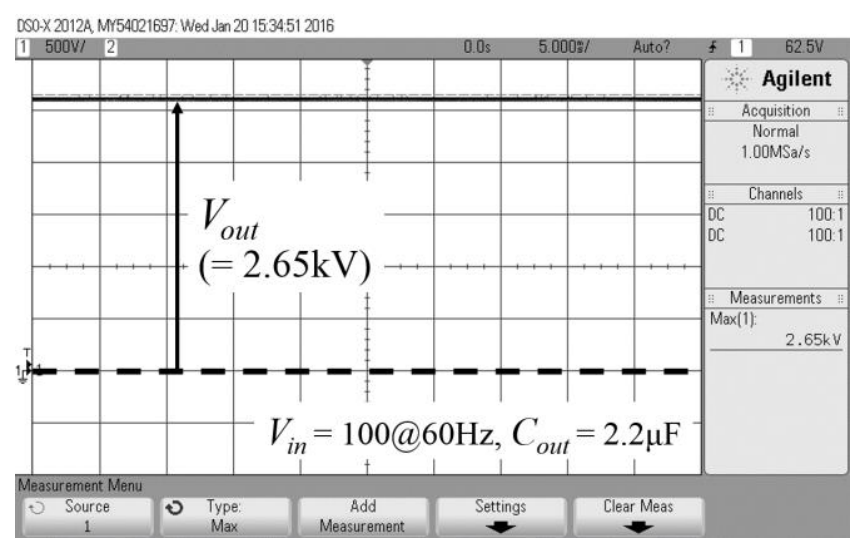

(a)

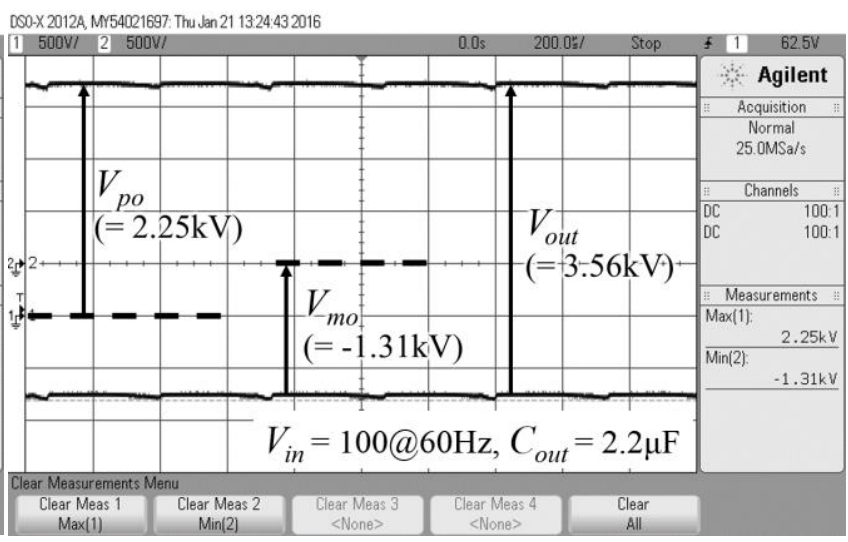

(b)

Fig. 3: Measured output voltages in a steady state; (a) conventional CWVM and (b) proposed voltage multiplier. 


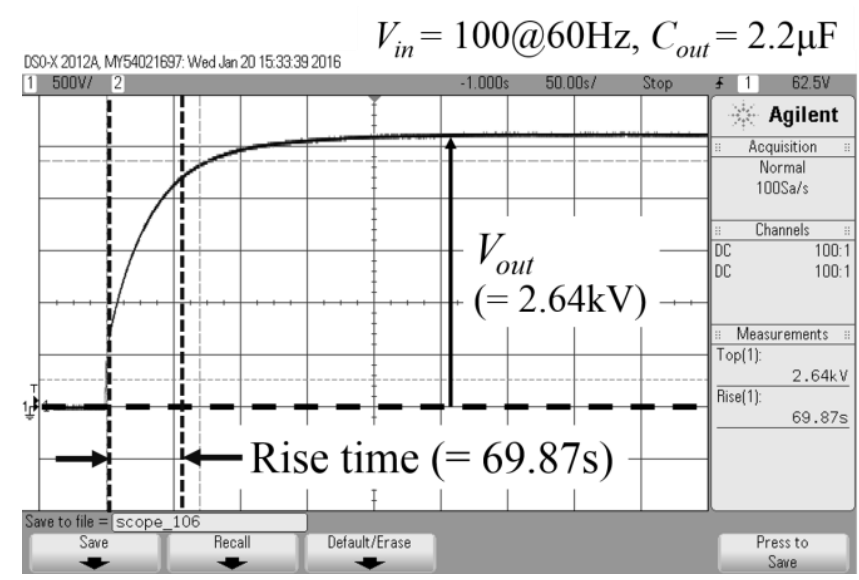

(a)

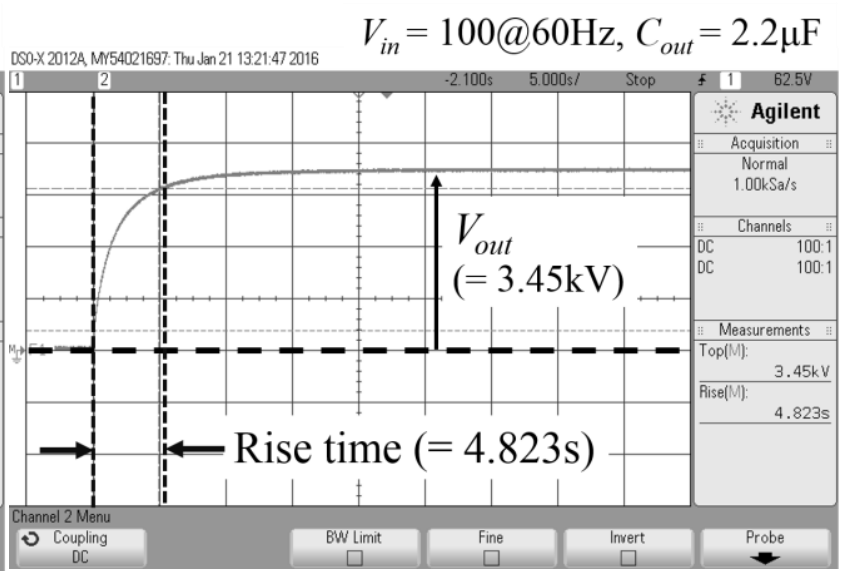

(b)

Fig. 4: Measured transient responses of output voltages; (a) conventional CWVM and (b) proposed voltage multiplier.

\section{Conclusion}

For non-thermal food processing by utilizing an underwater shockwave, a high step-up bipolar voltage multiplier using level shift drivers has been proposed in this paper. The validity of the proposed voltage multiplier were confirmed by the experiments. The experimental results showed the following results: (1) the output voltage of the proposed voltage multiplier is $910 \mathrm{~V}$ higher than that of the conventional CWVM and (2) the proposed voltage multiplier is more than 14 times faster than that of the conventional CWVM. Furthermore, the number of the circuit components of the proposed voltage multiplier is smaller than that of the conventional CWVM when more than $3.5 \mathrm{kV}$ is generated. From these results, the proposed voltage multiplier can achieve high voltage efficiency, high speed operation and small circuit components. Therefore, by using the proposed multiplier, underwater shockwave can be offer firster than the conventional method.

The detailed simulation and theoretical analysis of the proposed multiplier are left to a future study.

\section{Acknowledgement}

This work was supported by Urakami Foundation for Food and Food Culture Promotion.

\section{References}

[1] C.H. Zhang, T. Namihira, T. Kiyan, K. Nakashima, S. Katsuki, H. Akiyama, H. Ito, and Y. Imaizumi. Investigation of shockwave produced by large volume pulsed discharge under water. Proc. of IEEE Pulsed Power Conf. 2005, pp. 1377-1380.

[2] Y. Miyafuji, K. Shimojima, S. Tanaka, K. Naha, T. Aka, H. Maehara, and S. Itoh. Development of the pressure vessel for manufacturing the rice-powder using the underwater shock wave. Proc. of ASME 2011 Pressure Vessels and Piping Conf. 2011, pp. 53-56.

[3] A. Lamantia, P. Maranesi, and L. Radrizzani. The dynamics of the Cockcroft-Walton voltage multi-plier. Proc. of IEEE Power Electronics Specialists Conf. 1990, pp. 485-490.

[4] S. Iqbal. A hybrid symmetrical voltage multiplier. IEEE Trans. Power Electronics. 2014, 29 (1): 6-12.

[5] K. Eguchi, S. Pongswatd, S. Terada, and I. Oota. Bipolar high voltage multiplier with symmetrical structure. Proc. of Applied Mechanics and Materials. 2014, pp. 173-177.

[6] K. Eguchi, K. Abe, S. Terada, and I. Oota. A parallel-connected high voltage multiplier for non-thermal food processing. Proc. of the ICEECE 2015 Electrical Electronics and Civil Engineering Conf. 2015, pp. 14-18. 